\title{
Comportamiento de la repitencia universitaria: un caso de estudio
}

\section{University repetition behavior: a study case}

Elvira Moré Polanco Universidad de La Habana, Cuba celene@instec.cu https://orcid.org/0000-0003-2811-407X

Celene Maceira de Armas

Universidad de La Habana, Cuba https://orcid.org/0000-0002-7418-942X

Revista Cumbres Vol.7 №2

Versión electrónica ISSN 1390-3365 http://investigacion.utmachala.edu.ec/revistas/index.php/Cumbres http://doi.org/10.48190/cumbres.v7n1a5 


\title{
RESUMEN
}

El objetivo del presente trabajo es exponer cuál ha sido el comportamiento de la repitencia en el Instituto Superior de Tecnología y Ciencias Aplicadas (InSTEC) desde el curso 2014-2015 hasta el curso 2019-2020. Metodológicamente se utilizó la investigación de tipo observacional; desarrollada en un campo retrospectivo, con mediciones transversales y aplicando métodos de la estadística descriptiva, y la estadística no paramétrica. Se estudió una subpoblación conformada por todos los repitentes del período que se analiza según el registro en la secretaría docente del instituto. Se determinó que existe un pobre aprovechamiento de la repitencia, esta es mayor en el plan de estudios actual que en el anterior. No se aprecia diferencias entre las carreras que se estudian en la facultad y la repitencia se encuentra concentrada en los primeros años.

Palabras clave: educación superior, deserción, repitencia, calidad.

\begin{abstract}
The objective of the present work is to expose which behavior of the repetition has been in the Superior Institute of Technology and Applied Sciences (InSTEC) from the course 2014-2015 until the course 2019-2020. Methodologically was used in the investigation of observational type; developed in a retrospective field, with traverse mensuration and applying methods of the descriptive statistic, and the non-parametric statistic. A subpopulation was studied conformed by all the repeaters of the period that is analyzed according to the registration in the educational secretary of the institute. It was determined that there is poor use of the repetition that exists, this is greater in the current study plan than in the previous one. There is no apparent difference in repetition between the careers that are studied in the faculty and are concentrated in the first years of the careers
\end{abstract}

Keywords: higher education, desertion, repetition, quality.

\section{Cumbres}




\section{INTRODUCCIÓN}

Según la UNESCO los momentos actuales el bienestar de las naciones está estrechamente vinculado a la calidad y el alcance de sus sistemas e instituciones de enseñanza superior, autores como Cáceres y Ortiz consideran que la repitencia constituye un indicador de la calidad de la educación superior que tiene incidencia en su tasa de éxito o fracaso. Permite conocer las deficiencias ocurridas en los procesos de orientación, transición, adaptación o promoción de estudiantes. (Cáceres, Álvarez, \& Ortiz, 2014)

La problemática de las bajas y la repitencia en las Instituciones de Educación Superior latinoamericanas es percibida como fenómenos no deseables que han sido enfrentados en cada una de las etapas de su desarrollo con políticas y estrategias nacionales e institucionales orientadas a disminuir su impacto negativo en lo individual, lo institucional y lo social (Passailaigue y col, 2014).

Teniendo en cuenta la repercusión que esta problemática tiene para el desarrollo de la sociedad, es importante intensificar las investigaciones y estudios sobre el tema y proponer acciones que contribuyan a minimizarlo. (Cáceres, Álvarez, \& Ortiz, 2014) (Álvarez, Callejas, Griol, \& Durán)

La repitencia es una problemática multisectorial, multicausal y multifactorial en el que participan múltiples variables que responde a temporalidades iguales o diferentes, con incidencias en el ámbito personal, familiar, académico social, económico, entre otros. Todo esto permite considerar a la repitencia como un fenómeno complejo que debe ser estudiado en varias etapas que permitan dilucidar los aspectos involucrados con el fin de reorientar los procesos mediante medidas correctivas y estrategias que aminoren su impacto en las personas y contribuyan a elevar la calidad del sistema educativo universitario. (Ayala \& Cáceres, 2019) (Ajitimbay, Procel, \& Silva, 2019)

Se han realizado diferentes estudios relacionados con la repitencia. Algunos teóricos hacen referencia a diferentes modelos explicativos como son: el de Holland, Vincent Tinto, Bean y Metzner, Levy-Garboua, el psicopedagógico, estructuralista, sociológico y los que permiten los análisis econométricos de supervivencia. (Jiménez G., 2016) (Pérez, 2017) (Mosqueta, Capó, \& Jiménez, 2018).

Otros estudios abordan la temática en un determinado contexto universitario. Estos últimos están orientados a identificar los factores que intervienen en la repitencia estudiantil que pueden ser de diferentes tipos culturales, demográficos, económicos, sociales, académicos, pedagógicos, psicológicos e institucionales. En estas investigaciones se vincula el estudio de la repitencia con la realización de acciones correctivas. Su propósito es el de disminuir la repitencia estudiantil. Para ello se identifican dentro de la investigación las etapas de diagnóstico, análisis de las causas y la formulación de propuestas de mejora. (Loyola I., 2018) (Ajitimbay, Procel, \& Silva, 2019) (Ayala 
\& Cáceres, 2019) (Carrión, Céspedes, Suárez, Guerrero, \& Espinosa, 2019) (Guamán, Salazar, \& Cáceres, 2019) (Álvarez, Callejas, \& Griol, 2020)

La propuesta de mejoras, no garantiza por si sola la disminución de la repitencia estudiantil. Es necesario que estas mejoras se implementen, se les dé seguimiento, se evalúen y se sistematicen. Es un proceso que debe estar liderado por la dirección del Centro de Educación Superior con la participación de todo el personal docente o no docente que se encuentre implicado.

El estudio de la repitencia en el InSTEC tiene su origen en algunas interrogantes que se plantean los profesores del instituto ¿Cómo es el comportamiento de la repitencia?; ¿Qué se puede hacer para disminuirla? ¿Se ha incrementado la repitencia con el nuevo plan de estudios?, ¿Vale la pena dar la repitencia a un estudiante que está a punto de causar baja por dificultades académicas?

Para dar respuesta a estas interrogantes, se realiza un estudio preliminar de la misma. Este estudio tiene como objetivo proponer una base de referencia para abordar de una forma científica y estratégica la repitencia universitaria.

Este abordaje de la repitencia es posible en el contexto de las universidades cubanas donde las preocupaciones se centran en investigaciones sobre repitencia y las bajas en los cursos diurnos, las estrategias de permanencia en la universidad cubana, y la relación de esta con el pleno acceso y las transformaciones curriculares. (Cánova, Cruz, \& Vecino, 2016)

Desde el 2017 el Ministerio de Educación Superior sigue la metodología de gestión estratégica por objetivos y resultados, basada en valores y orientada a procesos. (Gónzalez, García, Fernández, \& González, 2016)

La planificación estratégica universitaria se alinea con el plan para el desarrollo del país hasta 2030, en todos sus ejes y sectores estratégicos. Entre las directrices de cambio y el proceso de transformaciones de la educación superior se encuentran las que se orientan al perfeccionamiento de la formación de pregrado: elevación de la eficiencia y a la contribución a los cambios del modo de dirección y la introducción de nuevos métodos de gestión y administración. Siendo la permanencia una prioridad en la gestión del proceso de formación del profesional. (Cánova, Cruz, \& Vecino, 2016) (Díaz-Canel \& Alarcón, 2020)

En este trabajo se presentan los resultados obtenidos en la primera fase de investigación orientada a dar respuesta a las siguientes interrogantes:

¿Cuál ha sido el comportamiento de la repitencia en el InSTEC en el período de 2015 a 2020 ?

¿Cuál ha sido el aprovechamiento de la repitencia 2015-2019?

¿Existen diferencias en el comportamiento de la repitencia por año?

$¿$ Existen diferencias en el comportamiento de la repitencia entre las diferentes carreras que se estudian en el instituto?

¿Cuál ha sido la incidencia del plan de estudios actual (plan E) en la repitencia con respecto al plan de estudios precedente (plan D)? 


\section{MATERIALES Y MÉTODOS}

Para el estudio de la repitencia en el InSTEC se propone un modelo que vincula la investigación a la estrategia de la organización. El proceso de estudio consta de cuatro fases: diagnóstico, análisis causal, intervención, evaluación de impactos y resultados. (Figura 1)

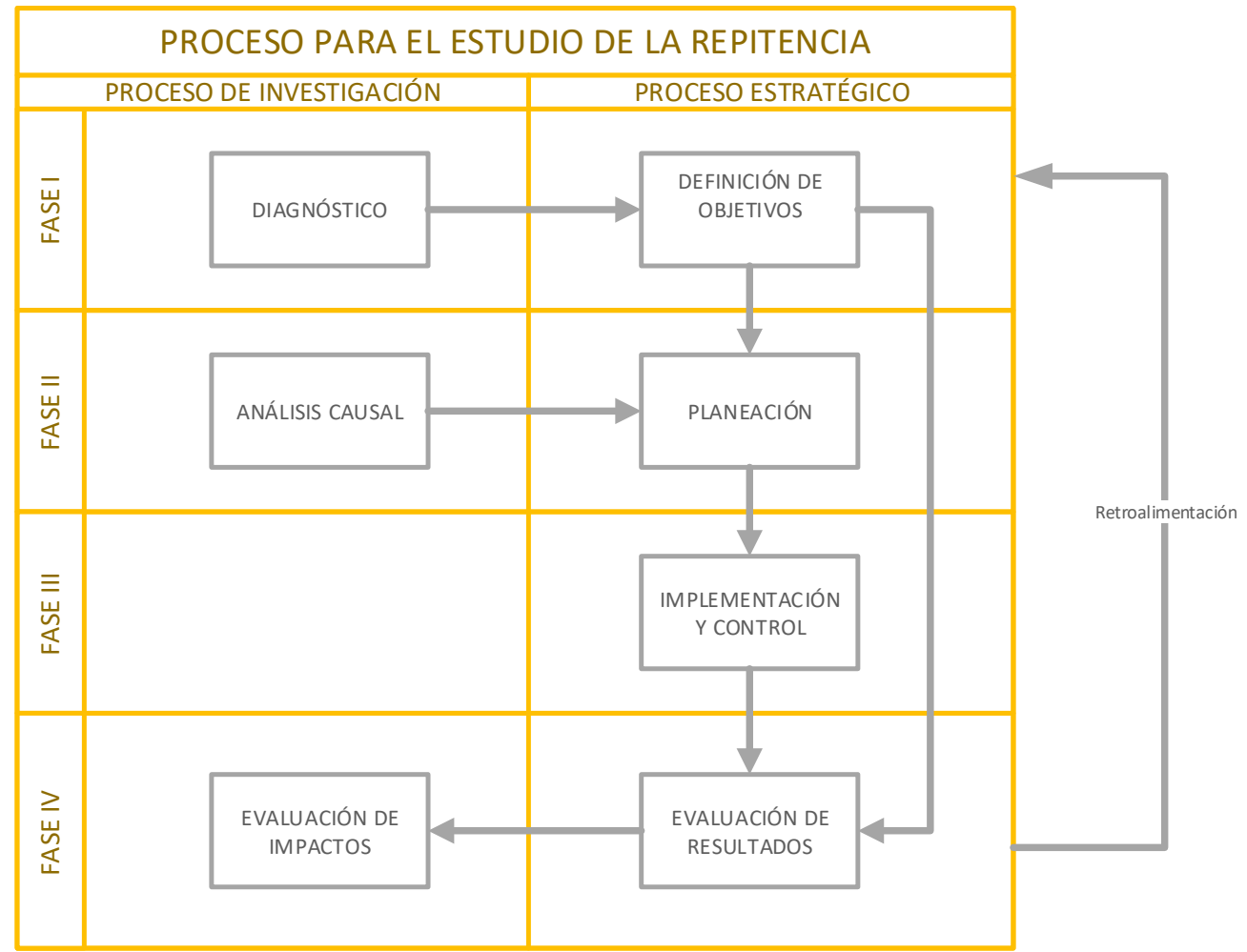

Figura 1. Proceso para el estudio de la repitencia (Elaboración propia)

La fase de diagnóstico (I) dirigida a conocer cómo se manifiesta la problemática de la repitencia en el instituto y la definición de objetivos por parte de la dirección del centro.

La fase exploratoria (II) orientada a identificar y analizar las causas de la repitencia y a partir de su conocimiento formular planes de acción por parte de la dirección.

La fase de intervención (III) encaminada a la implementación, seguimiento y control de las medidas propuestas.

La fase de cierre (IV) enfocada en evaluar los resultados, los impactos que fueron obtenidos, sistematizar las experiencias e identificar puntos de mejora. En la Resolución del Ministro de Educación Superior No. 120 de 2010 
Reglamento de Organización Docente de la Educación Superior, se establecen las normas relacionadas con la repitencia de un año académico, en correspondencia con el tipo de curso donde se encuentre matriculado el estudiante. (MES), 2010.

La repitencia se define como la acción de cursar reiterativamente una actividad docente, sea por mal rendimiento del estudiante o por causas ajenas al ámbito académico" (González Fiegehen, 2005, p.157) en (Guamán, Salazar, \& Cáceres, 2019).

Para el cumplimiento de los objetivos planteados se realiza una investigación no experimental diseñada como un estudio longitudinal con evolución de grupo (Hernández, 2003) que tiene como objeto la subpoblación de estudiantes que repiten un año en los cursos comprendidos entre 2014 - 2015 hasta el curso 2019-2020.

Se definen como variables de estudios la cantidad de estudiantes repitentes, el año que repiten, la carrera que estudian, los que causan baja y los repitentes que se gradúan.

Se presenta cómo han evolucionado los índices de repitencia, de otorgamiento desde el curso 2015 -2016 hasta el curso 2019-2020; y el aprovechamiento de la misma desde el curso 2015 -2016 hasta el curso 2018-2019.

El índice de repitencia es el porcentaje de estudiantes que repiten la/las asignaturas durante el siguiente período académico, es decir, no han aprobado la/las materias y deben cursarlas por segunda vez, se calcula como:

$$
I R=\frac{\text { repitentes }}{\text { matriculados }}
$$

Donde los repitentes es el número de estudiantes reprobados rematriculados en el mismo año académico en el siguiente curso, al no lograr promover y los matriculados hacen referencia al total de estudiantes matriculados en el período (t).

El índice de otorgamiento de la repitencia es el porcentaje de estudiantes que se les autoriza la repitencia por parte de la institución con respecto a los no promovidos en el curso anterior, se calcula como:

$$
I R O=\frac{\text { repitentes }}{\text { no promovidos }}
$$

Donde los no promovidos hacen referencia al total de estudiantes que son posibles bajas del curso anterior al que se analiza (t-1).

El índice de repitentes graduados es el porcentaje de estudiantes repitentes que se gradúan, con respecto a la matrícula del periodo que se analiza y se calcula como:

$$
I R G=\frac{\text { graduados }}{\text { repitentes }}
$$


Donde los graduados es el número de estudiantes que fueron repitentes y se graduaron en el curso que se estudia.

El índice de permanencia es el porcentaje de estudiantes repitentes que promueven de año, con respecto a la matrícula del periodo que se analiza, se calcula como:

$$
I R P=\frac{\text { promovidos }}{\text { repitentes }}
$$

Donde los promovidos es la cantidad de repitentes que promueve el curso que se analiza.

Para realizar este estudio se consultan las bases de datos de la secretaría docente del InSTEC Se emplean las tablas de frecuencias, y los estadígrafos de tendencia central y de dispersión para describir el comportamiento de la repitencia por años y por curso académico (McClave \& George, 1988), (InSTEC, 2020).

Se utiliza la comparación para conocer como se ha comportado la repitencia en las diferentes carreras del InSTEC.

Para conocer si ha aumentado la repitencia en el plan de estudios E con respecto al plan de estudios D se estudiaron cuatro cohortes, dos de los grupos que cursaron primero, segundo y tercer año con el plan de estudios D entre los cursos 14-15 y 15-16 y dos de los grupos que cursaron el plan de estudios E entre 17-18 y 19-20.

La prueba estadística empleada fue la de Chí cuadrado (Siegel, 1974). Para el cálculo de Chí cuadrado se utilizó la ecuación 1 y se consideró un nivel de significación de un $1 \%$.

$$
X^{2}=\sum_{i=1}^{2} \frac{\left(o_{i}-e_{i}\right)^{2}}{e_{i}}
$$

\section{RESULTADOS Y DISCUSIÓN}

Comportamiento de la repitencia en el InSTEC desde el curso 2015-2016 hasta el curso 2019-2020.

El Instituto Superior de Tecnologías y Ciencias Aplicadas es un instituto adscrito a la Universidad de La Habana, en él desarrolla la preparación universitaria de las carreras de Ingeniería en Tecnologías Nucleares y Energéticas, Licenciatura en Física Nuclear, Licenciatura en Radioquímica y Licenciatura en Meteorología. (Consejo de Ministros, 2016). 
Desde el curso 15-16 al curso 19 -20 en el InSTEC se matriculan como promedio por curso académico 370 estudiantes, de ellos repiten aproximadamente 22 lo que representa un $6 \%$ con respecto a la matrícula.

Desde el curso 15-16 hasta el curso 19-20 se aprecia una tendencia al aumento de las bajas y las repitencias. En los últimos cursos 18-19 y 19-20 se duplicaron las bajas con respecto a los cursos anteriores; y se les otorgó la repitencia al $50 \%$ de los estudiantes que ocasionarían baja. La cifra fue inferior en los cursos 15-16, 16-17 y 17-18. Ver Figura 2

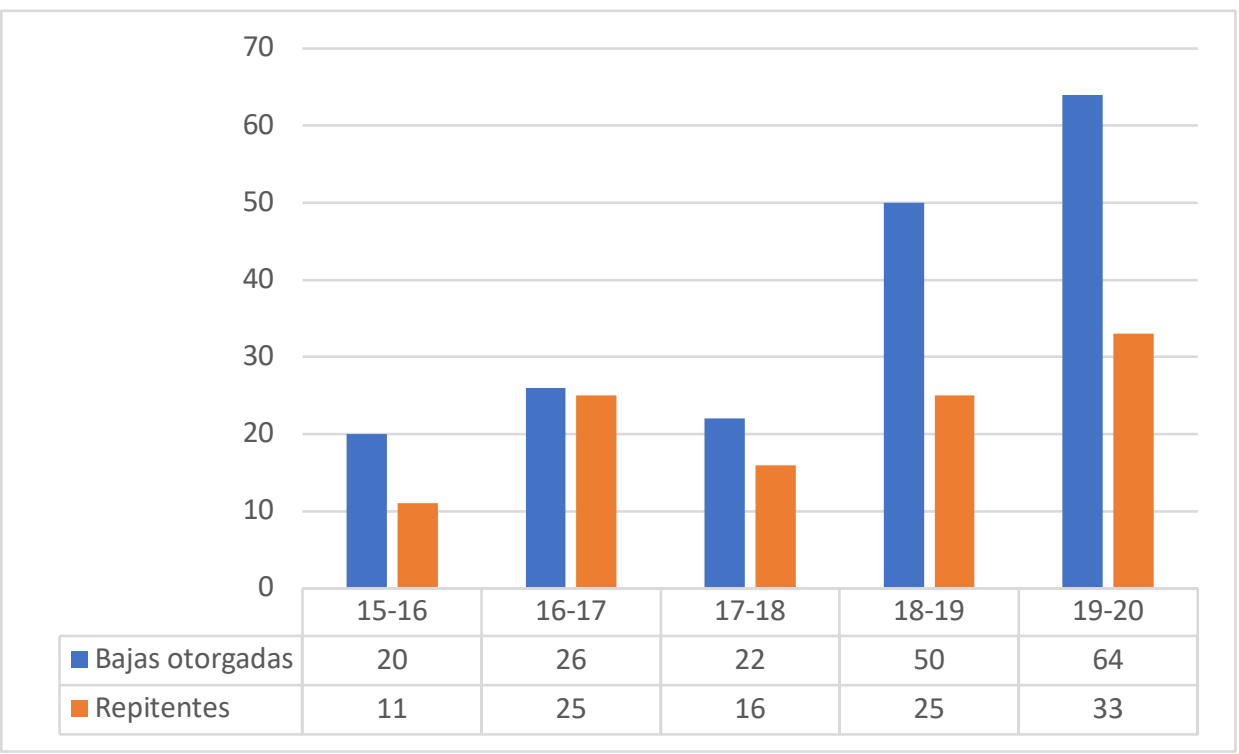

Figura 2. Bajas y repitentes por curso académico. (Elaboración propia)

En el período analizado se puede observar que existe una tendencia hacia el aumento de la repitencia, una disminución en cuanto al otorgamiento de la repitencia y la permanencia. Figura 3
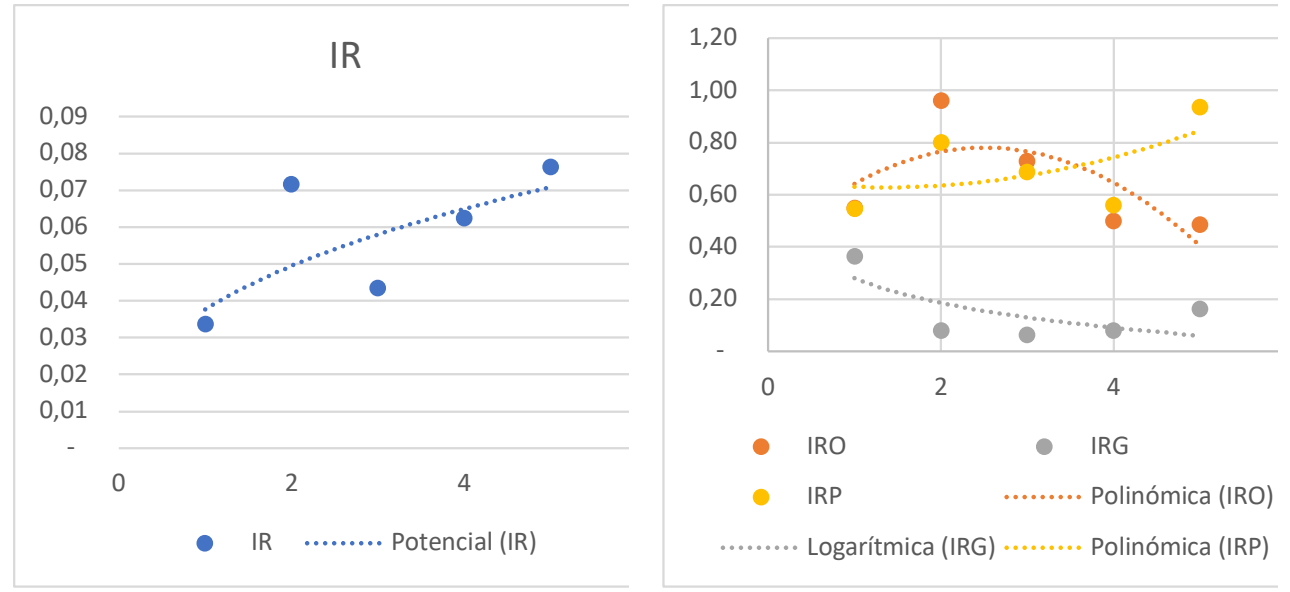

Figura 3. Comportamiento de la repitencia. (Elaboración propia) 
En el comportamiento de la repitencia con respecto a la matrícula se observa la existencia de dos máximos locales en los cursos 16-17 y otro en el curso 19-20, a este último le corresponde el mayor índice de repitencia de todos los cursos con un 7,64 \%. La relación de estudiantes repitentes que desertan tiene un valor mínimo en el curso $16-17$ con un 3,4\%. Se observa que a partir del curso 16-17 existe una tendencia hacia el aumento de deserción de los estudiantes repitentes. Ver Figura 4

En el curso 15-16 se obtuvo el mayor porciento de repitentes graduados un $36,4 \%$, en los cursos posteriores este porciento ha sido notablemente inferior, lo que evidencia que existe un pobre aprovechamiento de la repitencia por parte de los estudiantes. En el curso 19-20, se graduó el 84 \% de los estudiantes que solicitaron repitencia. Aunque este porciento es superior a los obtenidos en los cursos 16-17, 17-18, y 18-19 es bastante elevado. ya que curso 17-18 se obtuvo el menor porciento de graduados con respecto a las repitencias otorgadas. Ver Figura 5

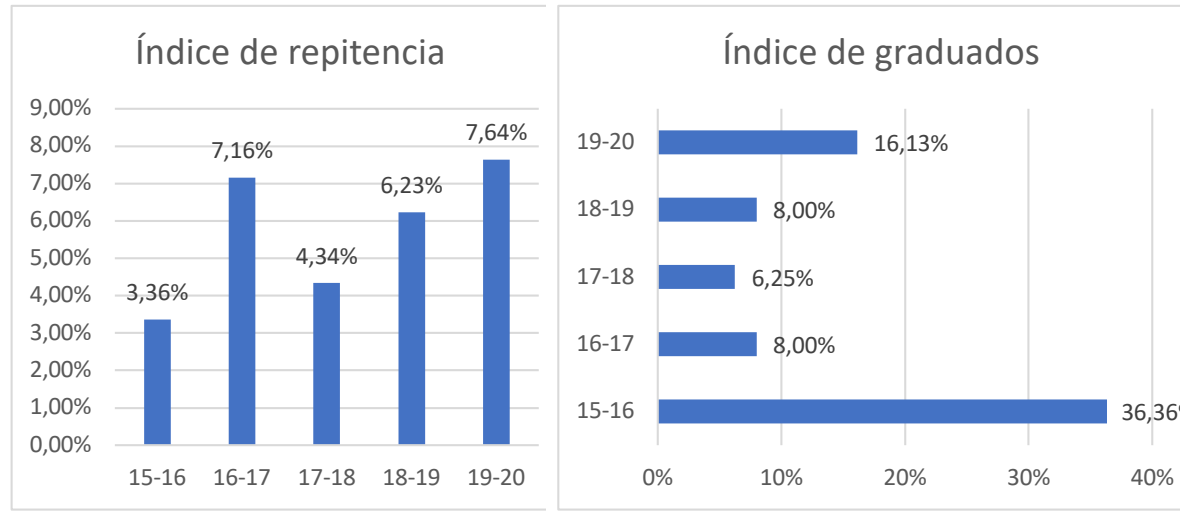

Figura 4. Comportamiento de la repitencia. (Elaboración propia) Figura 5. Comportamiento de graduados que repitieron en la carrera. (Elaboración propia)

Un estudio realizado por el Centro de Estudios para el perfeccionamiento de la educación superior en la Universidad de La Habana en tres carreras de siete instituciones universitarias cubanas consideraron como bajo un $4 \%$ de repitencia En el caso que se presenta con excepción del curso 15 -16, en los otros cursos estudiados el índice de repitencia es superior a este valor por lo que no se considera bajo. (Almiñas, y otros, 2021)

En los cursos estudiados se evidencia un comportamiento exponencial descendiente de la repitencia por año. El año que más repitencia tiene es primero con un $21 \%$, le sigue segundo año con un $8 \%$ y tercer año con un 2 \%. La repitencia en cuarto y quinto año son prácticamente cero. Ver Figura 6 
Primer año es el que más repitentes tiene el $65 \%$, en este año se encuentra al menos la mitad de las repitencias. Entre primero y segundo año se acumula el $91 \%$ del total de repitentes en el período analizado.

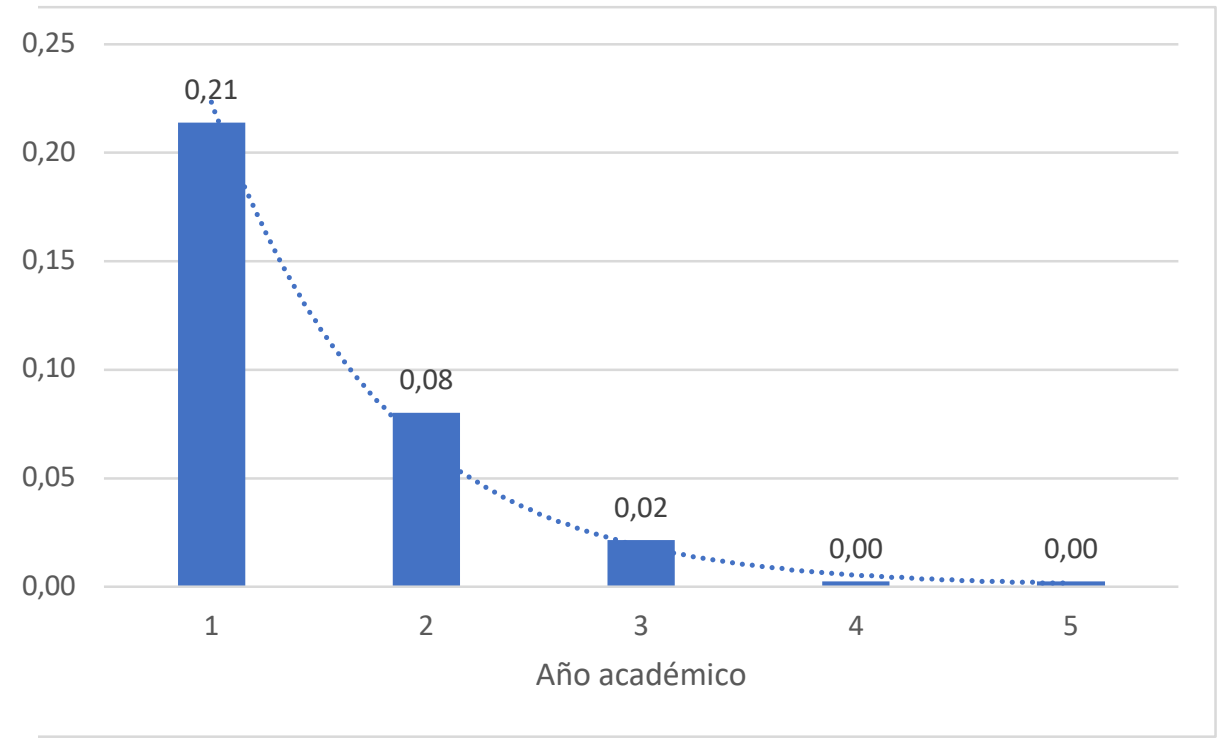

Figura 6. Índice de repitencia por año. (Elaboración propia)

Este resultado coincide con lo planteado por otros autores como Montenegro \& Taco, Allauca (2012) de la Universidad Central del Ecuador, ellos identificaron que en las facultades de Arquitectura y Administración carrera de Administración Pública, que la tasa de deserción y repitencia estudiantil, se presenta con un alto porcentaje en los primeros años (75\%). (Passailaigue, Amechazurra, \& Galarza, 2014)

Comparación de la repitencia entre las carreras del InSTEC

Entre las carreras del instituto en el periodo 2015-2020 el departamento que más repitencias ha tenido es el de Ingeniería Nuclear con un $8 \%$ de repitencia con respecto a la matrícula, las demás carreras Física Nuclear, Radioquímica y Meteorología presentan una repitencia que se encuentra entre un 5\% y un $6 \%$. Ver Figura 7

Como se puede observar el comportamiento de la repitencia es similar en las carreras de licenciatura, pero puede diferir con la repitencia de la carrera de ingeniería, este resultado se verifica con la prueba de X2.

Según los resultados de esta prueba se decide que no existen suficientes datos que permitan afirmar de que son diferentes las repitencias entre la carrera de ingeniería y las licenciaturas en el periodo analizado. 


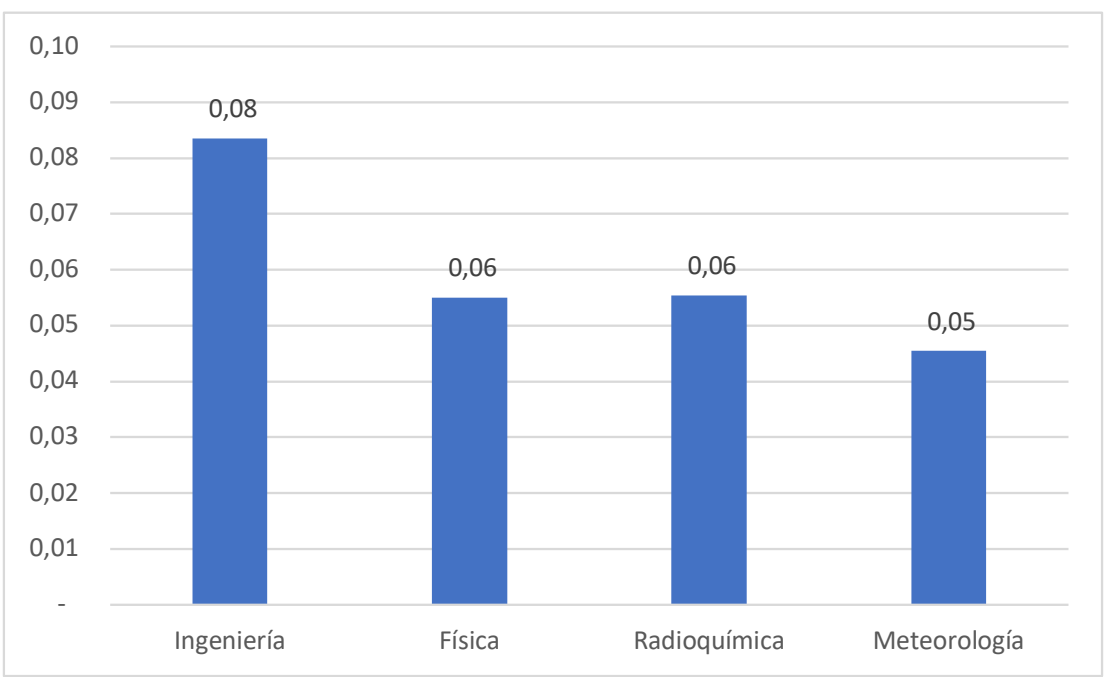

Figura 7. Índice de repitencia. (Elaboración propia)

Las carreras que más repitencias otorgan son las de Ingeniería Nuclear, le siguen Meteorología, Física Nuclear y Radioquímica en ese orden. En cuanto al número de repitentes que se gradúan se observa en primer lugar Meteorología con un $11 \%$, le sigue Ingeniería Nuclear con un $8 \%$ y por último Radioquímica y Física con un 5\% y 4 \% respectivamente. Según la permanencia en primer lugar se encuentra la carrera de Radioquímica seguida de Ingeniería, Física y Meteorología, respectivamente. Ver Figura 8

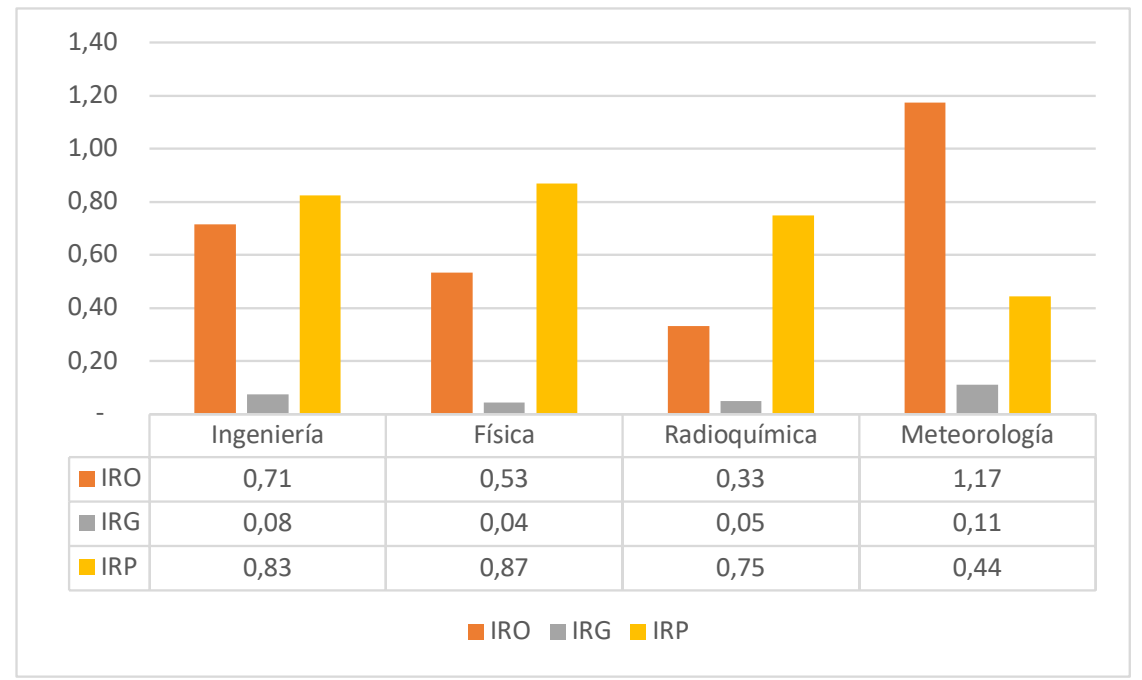

Figura 8. Comparación de índices entre carreras. (Elaboración propia) 


\section{Diferencia de la repitencia entre los planes de estudios D y E}

Después del proceso de cambios realizados a partir del plan de estudio $\mathrm{D}$, se implementa el plan de estudio E, primeramente en la carrera de Meteorología durante el curso 17-18, y se introdujo en las otras tres carreras posteriormente en el curso 18-19. Uno de los beneficios fue, que se redujo el tiempo de duración de las mismas, porque el ciclo es de 4 años.

Para constatar si existe diferencia en la repitencia entre los planes de estudio D y E se realizó la prueba de Chi cuadrado.

El valor obtenido de Chi cuadrado fue de 24.2. El resultado obtenido de esta prueba permite afirmar que la repitencia en el plan de estudios E es superior a la del plan $\mathrm{D}$ en el período estudiado.

\section{CONCLUSIONES}

En el país se tiene la preocupación por garantizar que todos los graduados de la enseñanza media, tengan asegurado su acceso al nivel superior. Pero es importante lograr la permanencia y egreso en esta enseñanza, para ello se establecen normas y resoluciones que permiten el cumplimiento de este proceso, como es la repitencia. Fenómeno complejo que debe ser abordado de forma proactiva desde una perspectiva científica y estratégica.

En el InSTEC durante el periodo 2015- 2020 se aprecia un aumento de la repitencia, una disminución de su otorgamiento y la permanencia. Desde el curso 15-16 y hasta el curso 19-20 se observa una disminución en el aprovechamiento de la repitencia. Los primeros años de las carreras son los que más aportan a la repitencia en el instituto. No existen diferencia entre la repitencia en las cuatro carreras que se estudian. Se observa un aumento de la repitencia en el plan de estudio E con respecto al D.

\section{RECOMENDACIONES}

A partir de los resultados obtenidos en esta primera etapa de la investigación se recomienda:

1. Que se proponga en la estrategia del InSTEC un objetivo orientado a disminuir las bajas y la repitencia.

2. Que se presente un proyecto que permita el estudio de las causas que motivan el aumento de la repitencia en el plan de estudios E, las repitencias y bajas en los primeros años de la carrera y el pobre aprovechamiento de la repitencia en el instituto. 


\section{REFERENCIAS BIBLIOGRÁFICAS}

Ajitimbay, C., Procel, A., \& Silva, L. (2019). Factores que intervienen en la repitencia estudiantil: caso escuela de contabilidad y auditoría. UNIVERSIDAD, CIENCIA Y TECNOLOGÍA, 18-24.

Almiñas, J., Galarza, J., Romero, B., Gómez, S., Vargas, A., \& De Armas, R. (12 de Noviembre de 2021). Acercamiento a la problemática de la repitencia y las bajas en la Educación Superior en el marco de la evaluación de la eficeincia académica: su magnitud y principales factores de influencia en carreras seleccionadas. Obtido de http://biblioteca.clacso.edu.ar/gsdl/collect/cu/cu-020/ index/assoc/D4497.dir/bajasyrepitencia.pdf

Álvarez, N., Callejas, Z., \& Griol, D. (2020). Factores que inciden en la deserción estudiantil en la carrera de Ingeniería Informática. Fuentes, 105- 126.

Álvarez, N., Callejas, Z., Griol, D., \& Durán, M. (s.d.). La deserción estudiantil en educación superior: s.o.s. en carreras de ingenería informática. VII CLABES. Séptima Conferencia Latinoamericana sobre el abandono en la Educación Superior. Córdoba.

Ayala, A., \& Cáceres, D. (2019). Factores Sociales, económicos y académicos que inciden en la repitencia de asignaturas de los estudiantes de la Facultad de Ciencias Económicas.

Cáceres, S., Álvarez, P., \& Ortiz, M. (2014). Deserción universitaria: la epidemia que aqueja a los sitemas de educación superior. Universidad y Sociedad.

Cánova, A., Cruz, L., \& Vecino, U. (2016). Apuntes de superación integral de los profesionales en instituciones de la educación superior. ATLANTE. Cuadernos de Educación y Desarrollo.

Carrión, E., Céspedes, E., Suárez, N., Guerrero, A., \& Espinosa, M. (2019). Factores que influyen en el resultado docente de estudiantes repitentes del primer año de medicina, Facultad Calixto García. Curso 2018- 2019. Edumed Holguín 2019. Holguín: Cuba.

Consejo de Ministros. (2016). Resolución. Cuba.

Díaz-Canel, M., \& Alarcón, O. (2020). Potencial humano, innovación y desarrollo en la planificación estratégica de la educación superior cubana 2012-2020. Educación Superior.

Gónzalez, M., García, J., Fernández, A., \& González, W. (2016). Mecanismos de gestión de la ciencia, la tecnología y la innovación en las universidades como herramienta indispensable para su avance. Universidad de La Habana.

Guamán, A., Salazar, \& Cáceres. (2019). Factores sociales, económicos y académicos que inciden en la repitencia de asignaturas de los estudiantes de la Facultad de Ciencias Económicas de la Universidad Central del Ecuador del período 20182019. Trabajo de investigación previo a la obtención del título de Ingeniero en Estadística. Quito.

Hernández, R. (2003). Metodología de la investigación 1. La Habana: Félix Varela. InSTEC. (2020). Base de datos de la secertaría docente. La Habana.

Jiménez, G. (2016). Reflexiones teóricas del fenómeno de repitencia abandono y deserción estudiantil. Pensamiento Actual, 261-269. 
Jiménez, L. (2016). Reflexiones teóricas de fenómeno de repitencia, prolongación, abandono y deserción de estudiantes universitarios. Pensamiento Actual.

Loyola, E. (2018). Repitencia estudiantil en la Universidad Politécnica Salesiana. Quito: Abya-Yala.

Loyola, I. (2018). ¿Por qué el estudiante repite en la Universidad Politécnica Salesiana? Em I. Loyola, Repitencia estudiantil en la Universidad Politécnica Salesiana (p. 131). Quito: Abya-Yala.

Martínez, J., Ortíz, Y., Pérez, H., Guevara, R., \& Ferrás, Y. (2020). Deserción estudiantil durante los primeros cinco años de la carrera Medicina. Electrónica Dr, Zoilo E. Marinello Vidaurreta.

McClave, J., \& George, P. (1988). Statics for business and economics. London: COLLIER MACMILLAN.

MES. (s.d.). Reglamento de organización. Cuba.

Mosqueta, Y., Capó, J., \& Jiménez, Y. (2018). Modelación para predecir indicadores académicos en la Educación Superior. Ciencia universitaria.

Passailaigue, R., Amechazurra, O., \& Galarza, J. (2014). La deserción y la repitencia en las instituciones de Educación Superior. Algunas experiencias investigativas en el Ecuador. Universidad y Sociedad, 102-107.

Pérez, P. (2017). Hacia un seguimiento proactivo de la repitencia estudiantil: un enfoque desde la esperanza matemática. Pertinencia Académica.

Roca, W. (2019). La repitencia en la educación superior, su entorno y consecuencia. Guatemalteca de Educación Superior, 75-90.

Siegel, S. (1974). Estadística no paramétrica. México: Trillas.

\section{Cumbres}

